\title{
The Space and the Physical Time Are Interdependent On Each-Other: The Numbers Associated With the Measurement of the Physical Time Are Macroscopic, Abstract and Deviation from Idealism
}

\author{
Prasenjit Debnath
}

PhD Student, NIT Agartala, India

\begin{abstract}
The space and the physical time are interdependent on each other. There are asymmetric distributions of space points in the Universe and because of that, there are asymmetries in resultant macroscopic times in different local areas (any local area has only even distribution of space points within the area, as a result, the resultant time is symmetric within the local area which means that the resultant time is same at every point within the local area but asymmetric between different local areas which means every local area has a unique resultant time). Similar reasoning can be put forward is that because the Universe has different local areas having symmetrical macroscopic resultant time within the local area and asymmetric resultant macroscopic times among different local areas, there are asymmetric distribution of space points in the Universe. In those many local areas, there are different resultant macroscopic times with different directions compared to $x$-axis which means that different macroscopic times sometime add each other and sometime cancel out each other to limit the real time (component along $x$-axis) to be within the psychological recognition range. Now, any local area can be as tiny as an atom or as big as more than a solar system or may be as large as a galaxy. The local area is that particular area where all space points are evenly distributed and because of that the macroscopic resultant time of that area is such that we can measure the same macroscopic resultant time in any particular point of that area. This is the macroscopic appearance of the physical time that can be recognizable by psychology. The resultant macroscopic time can be calculated by adding of all unit vector of time associated with each space point of a local area and finding out the real component part of the resultant macroscopic time along the $x$-axis which is the effective time movement of that local area. If for any local area consists of only vertically directed time vector, it implies that there is no real part of component of the physical time along the $x$-axis, thus no time flows in that area at least within the range of vision of psychology. If any local area consists of points with associated time vectors parallel to the $x$-axis, all unit vector of time associated with each space point will add up, and the resultant macroscopic time will only be increased along the x-axis. The bigger the local area, the more addition of unit time vectors associated with each point of that local area, the faster the macroscopic time flow in that local area. If the whole universe would be totally uniform in space point distribution and if the unit times are directed along the x-axis, the resultant macroscopic time vector would be infinitely fast at least on the psychological point of view. But there is in-built non-uniformity in space point distribution in the Universe so that the physical time slows down to a level that psychology can put a number on that and it makes ease and comfortable to go with along. Thus the asymmetry of the Universe puts limit to the physical time flow and time flows at different rate at the different local areas (having symmetry within the local area which can be termed as local area of symmetry) of the Universe.
\end{abstract}

Keyword: The space and the physical time, asymmetric distributions of space points, symmetrical macroscopic resultant time, unit vector of time, local area of symmetry

\section{Introduction and Theory}

The Earth is composed of many types of matter at different states, for example- solid, liquid and gas [1, 2]. If each atom (or might be a quark) can be treated as a point (elemental representation of macroscopic spatial dimensions such as length, width and height $[3,4]$ ) which does not have any macroscopic dimension of itself $[5,6]$. Thus, each atom has a unit time vector associated with it in a particular direction. And a slight variation is there with the direction of unit time vector of a gold atom compared to a silver atom. Any gold mine can be a local area of time symmetry if in that local area only pure gold is available. Any pond or river or sea or ocean can be a local area of water which has something other macroscopic time compared to gold mine. Also the magnitude of the resultant macroscopic time of ocean is way way larger than a gold mine simply because ocean has way way more $\mathrm{H}_{2} \mathrm{O}$ atoms compared to the Gold atoms in the gold mine [7, 8]. Oxygen $\left(\mathrm{O}_{2}\right)$ has different macroscopic time compared to Nitrogen $\left(N_{2}\right)$ in the air. Similar thing applies to all other gases that form our atmosphere. Now, what is the macroscopic resultant time for the air itself? It is addition and subtraction of unit time vectors of all molecules (or all local areas of symmetry, if any) in the air. Because the resultant times of each type of molecules (or local areas) are at different directions, the resultant macroscopic times are subtracted way more than added by the same type of molecules. It is because the variety of molecules is more than similarity of molecules. If the air would be of same type of molecule, the resultant macroscopic time would be faster than the air of present state (a mixture). Because there are huge variety of molecules in the solid and liquid in the Earth makes the resultant macroscopic times of solid and liquid in the earth slower and slower. The variation of matter states in the Earth such as solid, liquid and gas makes the resultant time of the Earth further slow down. Matter is nothing but 


\section{International Journal of Science and Research (IJSR) \\ ISSN (Online): 2319-7064 \\ Index Copernicus Value (2013): 6.14 | Impact Factor (2014): 5.611}

the space deformation, different types of matter has different degree of space deformations. There are very very less deformation of the space points in the free space. Also free space does not have that much variety of space points deformations compared to Earth. Thus the free space forms bigger local area than the local areas in the Earth. These are the reasons that the resultant macroscopic time runs faster in the free space compared to the Earth. If the Earth would be of more variety of atoms, the resultant macroscopic time would run slower than the present real Earth. If the Earth would be of more similar types of atoms (having less variety of different atoms), the resultant macroscopic time of Earth would be faster than the present real world. Thus, different space deformations made different variety of atoms. The more variety in an area (Earth), the further slowdown of the resultant macroscopic time is of that area. The highest deformation of space possible in the present state of the Universe is none other than Black Holes; thus time runs slowest possible inside the Black Holes. It is not unreasonable to assume that the unit time vectors associated with each point in the Black Holes are more towards the imaginary axis; thus the real component of it is very very lower in magnitude, the reason, the real time that psychology understands runs slower in the Black Holes $[9,10]$.

\section{Analysis of Unit Time Vectors Associated with Different States of Matter}

There are different states of matters such as solid, liquid and gas $[11,12]$. Gas is less compressed compared to liquid [13, 14]; thus gas has less space deformation compared to liquid. For a pure gas, the resultant macroscopic time vector is more horizontal compared to the resultant time vector of a pure liquid. For a pure solid, the resultant time vectors are more vertical compared to liquid and gas. Because the resultant time vector of a solid has less real part component compared to liquid of same amount, the resultant macroscopic time runs slower in solid compared to liquid of the same amount.



Figure 1: For a same amount of gas, atom's unit vector are more horizontal and thus the real time (x-axis component) is larger for pure gas compared to pure liquid and pure solid.

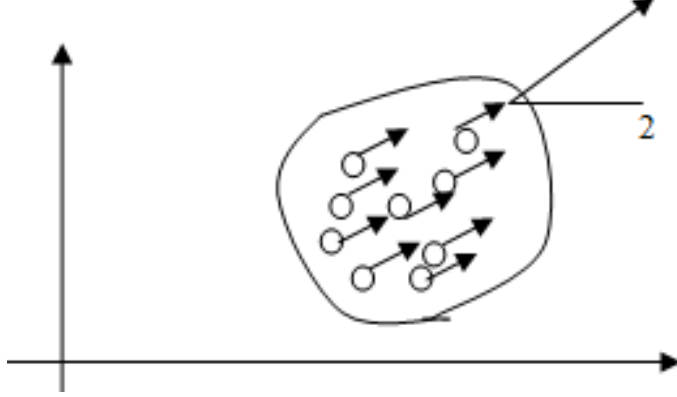

Figure 2: For a same amount of liquid, atom's unit vector are less horizontal and thus the real time (x-axis component) is lower for pure liquid compared to pure gas but larger compared to pure solid

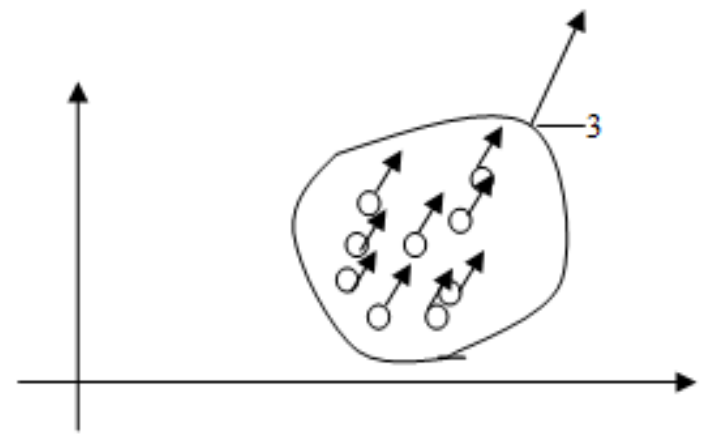

Figure 3: For a same amount of solid, atom's unit vector are least horizontal ( almost vertical) and thus the real time (xaxis component) is lowest for pure solid compared to pure gas and pure liquid

\section{How the Variety of Atoms Effect The Physical Time and the Gravity on Earth}

The Earth is composed of many types of matter at different states, for example- solid liquid and gas [15]. Each types of matter have different resultant time vectors. Because Earth is a mixture of many varieties of atoms $[16,17]$, the unit time vectors are scattered in an area of Earth $[18,19]$. Because the Earth is composed of mostly solid and liquid [20, 21] which has very very less real part component of unit time vector, the resultant macroscopic time of Earth runs slower than compared to the free space.

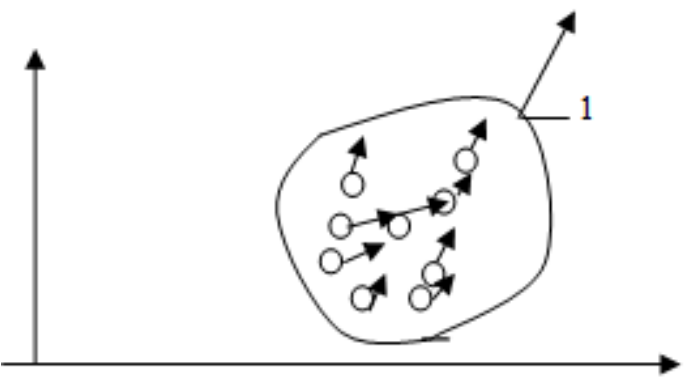

Figure 4: Real time (x-axis component of time) of Earth (1) is lower in Magnitude.



Figure 5: For a same amount space points in the free space, point's unit vector of time are more horizontal and thus the real time (x-axis component) (2) is larger compared to pure gas, pure liquid and pure solid.

Each space point is associated with its gravitational force. Thus any area of free space also has gravity and we also have individual gravity as a living being [22, 23]. But gravity is directly proportional to the degree of deformation of space. Because solids are highly deformed compared to liquid and gas of the same amount, solid has highest 


\section{International Journal of Science and Research (IJSR) \\ ISSN (Online): 2319-7064 \\ Index Copernicus Value (2013): 6.14 | Impact Factor (2014): 5.611}

gravitational force. Because gravity is a collective force [24, 25], it is effective with massive body and of long range [26, 27]. Because the Earth has variety in different types of atoms $[28,29]$, the gravity is not uniform everywhere as predicted by Newton's law of gravity. Also space-time curve also not uniform as predicted by Einstein's general theory of relativity. The following figure shows non-uniformity of space-time.



Figure 6: Einstein's Uniform space-time curvature

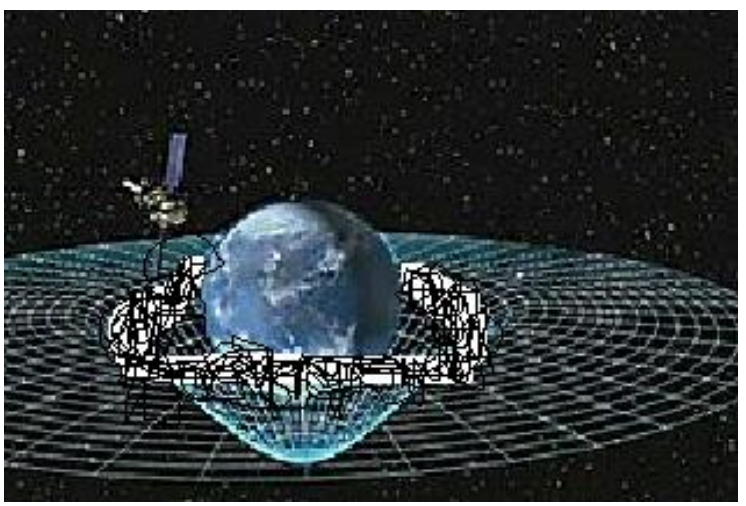

Figure 7: Non-uniform space-time curvature in reality.

\section{Conclusion}

There are asymmetric distributions of space points in the Universe makes the resultant macroscopic time limited in the Universe. If the Universe would be entirely uniform, the Universe would have infinite speed of time. The Black Holes have huge imaginary component of time and negligible real part, as psychology only understand the real part of time, the physical time runs too slow inside the Black Holes. Every space point has its own gravity and different types of atom have different strength of gravitational force. This is the reason Earth gravity is non-uniform all around which contradicts Newton's theory of gravity which tells us that the gravity is independent of the body of which it is made of. Also non-uniformity of gravity of Earth shows that the space-time curvature is non-uniform which contradicts Einstein's theory of general relativity. In reality, it is distorted curvature of space-time. Due to non-uniformity of Earth, the slowness of time is very very tiny compared to the free space and gravity variation is so less all around the Earth, we can safely ignore them on psychological ground. That is our trend of progress as we feel what to do with a 0.00000000000016 compared to 10,000 . With that set of mind, we can safely ignore the non-uniformity of space-time curvature and gravitational variation to serve our purpose. Because the calculations are not of the purest form, it is abstract and deviation from idealism which serves our purpose till date.

\section{Acknowledgment}

I cordially admire Dr. Aparna Nath, Associate Professor and my PhD Guide, The department of Physics, National Institute of Technology, Agartala, India, for the epitome of inspiration and motivation to write this particular paper with perfection and accuracy. I am extremely thankful to her from all possible help she made to write this paper. Also I am thankful to The Department of Physics of National Institute Of Technology Agartala (NIT Agartala) for proper conduct and coordination.

\section{References}

[1] Prasenjit Debnath, "Time and Space Are Mutually Inherent Property of Each Other, Where the Dependency of Time on Space Can Be Termed as Space-Time and the Dependency of Space on Time Can Be Termed as Time-Space" International Journal Of Science And Research, ISSN (online)- 2319-7064, volume 5 Issue 2, pp 1946-1948

[2] Brian Cox, Jeff Forshaw, "The quantum universe: everything that can happen does happen", Penguin Books, pp. 1-44.

[3] Stephen Hawking, "A Briefer History of Time”, Bantam Books, London, pp. 1-145.

[4] http://map.gsfc.nasa.gov/universe/uni_fate.html

[5] Stephen Hawking, "Black holes and Baby Universes and other essays", Bantam Press, London 2013, ISBN 978-0-553-40663-4

[6] Stephen Hawking, "The Grand Design”, Bantam Books, London 2011

[7] Stephen Hawking, "A Brief History of Time", Bantam Books, London 2011, pp. 156-157. ISBN-978-0-55310953-5

[8] Stephen Hawking, "The Universe in a Nutshell", Bantam Press, London 2013, pp. 58-61, 63, 82-85, 9094, 99, 196. ISBN 0-553-80202-X

[9] Stephen Hawking, "The Beginning of Time", A Lecture.

[10] Stephen Hawking, “Stephen Hawking's Universe: Strange Stuff Explained", PBS site on imaginary time.

[11] Stephen Hawking, "How to build a time machine", 27 April, 2010.

[12] Uno Ingard, K "Fundamental of Waves \& oscillations", Cambridge University Press. P. 38, ISBN-0-521-33957XOxford: The British Academy, 1999

[13]A. Zee, "Quantum Field Theory in a Nutshell", Princeton University Press, 2003

[14] Storrs McCall, "A Model of the Universe", Oxford: Clarendon Press, 1994

[15] Craig Callender, "Time, Reality and Experience", Cambridge, UK: Cambridge University Press.

[16] Craig Callender, "Thermodynamic Asymmetry in Time", The Stanford Encyclopedia of Philosophy (Spring 2002 Edition)

[17] Storrs McCall, "A Model of the Universe", Oxford: Clarendon Press, 1994 


\section{International Journal of Science and Research (IJSR) \\ ISSN (Online): 2319-7064}

Index Copernicus Value (2013): 6.14 | Impact Factor (2014): 5.611

[18]Robin Le Poidevin and Murray McBeath, “The Philosophy of Time" Oxford: Oxford University Press, 1993

[19] Newton-Smith, W.H., "The Structure of Time". London: Routledge \& Kegan Paul, 1980.

[20] Barry Dainton,'Time and Space", Ithaca: McGillQueen's University Press, 2001

[21] Robin Le Poidevin, "Questions of Time and Tense", Oxford: Oxford University Press, 1998.

[22] Nerhlich, Graham, "What Spacetime Explains". Cambridge: Cambridge University Press, 1994.

[23] Sklar, Lawrence, "Space, Time, and Space-time". CA: University of California Press, 1974.

[24] Whitrow, G., "The Natural Philosophy of Time". Oxford: Oxford University Press, 1961. (2nd edn., 1980.)

[25] S.W. Hawking, and G.F.R. Ellis, "The Large Scale Structure of Space-Time", Cambridge University Press, (1973).

[26] Stephen Hawking, "A stubbornly persistent illusion-The essential scientific works of Albert Einstein”, Running Press Book Publishers, Philadelphia, London 2011.

[27] Flynn, John L, "Time travel literature", on 29-09-2006

[28] Stephen Hawking, "The Theory of Everything", Jaico Books, pp. 1-110.

[29] Stephen Hawking, "The Illustrated A Brief History Of Time”, Bantam Books, pp. 1-21.

\section{Author Profile}

Prasenjit Debnath, born in Agartala, Tripura, India on $15^{\text {th }}$ of March 1979. He is pursuing a PhD degree in the Department of Physics in National Institute of Technology Agartala (NIT Agartala), India. 